\title{
Direct Visualization of Tumor Propagation by Cancer Stem Cells and Associated Tissue Responses
}

\author{
J.T. Myers ${ }^{1}$, J.D. Lathia ${ }^{2}$, A. Petrosiute ${ }^{1}$, Y. Othman ${ }^{1}$, K. Davis ${ }^{1}$, D. Barkauskas ${ }^{1}$, J.N. Rich ${ }^{2}$ and \\ A.Y. Huang ${ }^{1}$ \\ ${ }^{1}$ Division of Pediatric Hematology / Oncology, Department of Pediatrics, Case Western Reserve \\ University School of Medicine, WRB 6528, 2103 Cornell Road, Cleveland, OH 44106-7288 \\ ${ }^{2}$ Department of Stem Cell and Regenerative Medicine, Cleveland Clinic Foundation Lerner Research \\ Institute, Cleveland, $\mathrm{OH} 44195$
}

Human tumors commonly display heterogeneity within their neoplastic compartment, which may be derived from a combination of stochastic genetic copy number alterations and an epigenetic hierarchy that co-evolve over time [1]. The cancer stem cell (CSC) hypothesis represents an attractive model to describe many aspects of observed tumor behavior; however, the identity and functional impact of CSC remains controversial due in part to a lack of appropriate ex vivo experimental systems to fully capture the complex intercellular communication between tumor cells and surrounding tissue microenvironment - a critical feature in understanding processes associated with cancer development. Xeno-transplantation of human tumors, especially leukemic stem cells, into immune-deficient mice has supported the existence of CSC-enriched fraction with enhanced tumor formation capacity [2-5]; however, limited evidence exists for solid tumors. In this regard, commonly utilized whole-body in vivo imaging modalities, such as bioluminescence imaging, lacks the single-cell resolution necessary to discern subtle structural differences and cellular heterogeneity in the tumor stem cell niche environment. On the other hand, traditional high-resolution microscopic examinations of fixed tumor-infiltrating tissue specimen are devoid of the 3-dimensional tissue context or sequential evolution of tumor progression in the same tumor-bearing host. Recent new insights into in vivo tumor formation, neovascularization, metastasis and associated tissue / immune responses have come from studies involving the use of intravital 2-photon laser scanning microscopy (2P-LSM), a technique we and others have utilized over the past several years to visualize immune cell trafficking and interactions in different anatomical locations within anesthetized experimental mice [6-10]. This imaging modality allows deep tissue visualization $(>300 \mathrm{um})$ with single-cell resolution $(<1 \mathrm{um})$, thus enables direct observation of tumor and other cellular behavior in intact tissues at a spatial-time resolution suitable for tracking cellular migration, proliferation and interactions $[6,9-10]$.

We studied the role of CSC in the most prevalent primary malignant brain tumor, glioblastoma multiforme (GBM). GBM contains a cellular hierarchy with a sub-population $\left(\mathrm{CD} 133^{+}\right)$representing the self-renewing and tumorigenic GBM stem cells (GSCs). We fractionated GBM tumor samples into $\mathrm{CD}_{133^{+}}$and $\mathrm{CD} 133^{-}$populations, differentially labeled these cells with fluorescent reporter constructs so they can be distinguished from one another in vivo by fluorescence emissions under 2P-LSM. We observed that when transplanted into recipient nude mice, a single GSC was capable of tumor initiation and propagation in the brains of experimental mice. To directly test the relative tumorigenic potential of GSCs $\left(\mathrm{CD} 133^{+}\right)$and non-GSCs $\left(\mathrm{CD} 133^{-}\right)$, we interrogated paired tumor populations purified from primary human GBM tumor cells in competitive in vivo tumor growth studies as monitored by serial 2P-LSM through implanted cranial windows. Even though GSCs represented a small fraction of the initial cancer cell inoculum, serial 2P-LSM imaging shows that after 35 days, in vivo GBM tumor formation was driven exclusively by GSCs, but not non-GSCs. and the formed tumors displayed a cellular hierarchy (Figure 1). We also observed close relationship 
between metastasizing GSCs with neovascularization. Our data provide the first direct functional evidence that CSCs are responsible for tumor propagation in GBM, and the serial in vivo 2P-LSM imaging model provides an experimental platform for monitoring of therapeutic interventions and other manipulations aimed at targeting cancer stem cells and associated microenvironment.

Currently, our laboratory is also focused on understanding the role of tumor microenvironment in shaping immune repertoire towards tolerance, and developing strategies to modify tumor microenvironment for the generation of effective anti-tumor immunotherapy approaches. Such efforts aim not only at understanding dynamic tumor microenvironment in the periphery, the bone marrow, the lymph nodes and the CNS, but also on the associated immune responses within these organ systems. Some of these findings will also be highlighted at the Symposium.

References

[1] K. Anderson et al., Nature. 469 (2011) 356.

[2] J.M. Rosen et al., Science. 324 (2009) 1670.

[3] A. Colmone et al., Science. 322 (2008) 1861.

[4] L. Celso et al., Nature. 457 (2009) 92.

[5] S. Mendez-Ferrer et al., Nature. 466 (2009) 829-834.

[6] A. Huang, et al., Immunity. 21 (2004) 331.

[7] Huang, A.Y., Biol. Blood and Marrow Transpl. 13 (2007) 111.

[8] Miller, M.J. et al., Science 296 (2002) 1869.

[9] Mempel, T.R. et al., Nature 427 (2004) 154.

[10] Witt, C.M. et al., PLoS Biol. 3 (2005) 160.

[11] This work is supported by grants from the St. Baldrick's Foundation, Cancer Research Institute, the Dana Foundation, the Gabrielle's Angel Foundation, the Hyundai "Hope-on-Wheels" Research Award, and the Rainbow Fellow Research Award Program.

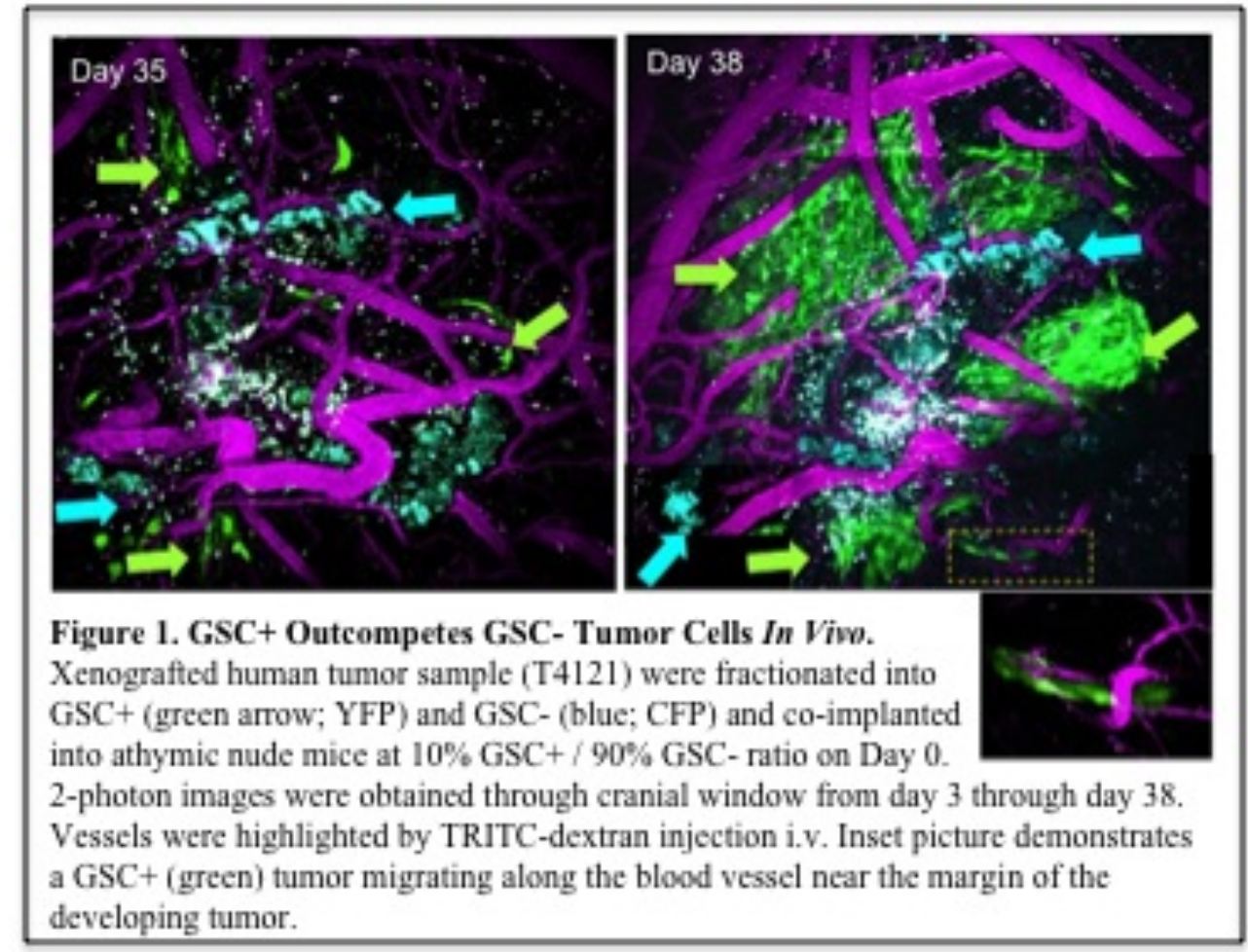

